\title{
La recomposición del espacio de actividad económica metropolitano. El Área Metropolitana de Valencia
}

\author{
Ana Gascón-Hernández ${ }^{1}$, Carmen Blasco-Sánchez ${ }^{2}$, \\ Francisco Juan Martínez-Pérez ${ }^{3}$, Julia Deltoro-Soto ${ }^{4}$ \\ ${ }^{12}$ Departamento de Urbanismo. ${ }^{4}$ Departamento de Ingeniería de la Construcción y de Proyectos \\ de Ingeniería Civil. ${ }^{12} 34$ Universitat Politècnica de València, España \\ E-mail: 1angasher@doctor.upv.es, ${ }^{2}$ mblasco@urb.upv.es, \\ 33jmartpe@urb.upv.es, ${ }^{4 j u d e l s o @ c s t . u p v . e s ~}$
}

\begin{abstract}
Resumen. Las áreas de actividad económica, en sus diferentes modalidades (producción, logística, comerciales, de dirección o negocio) pueden desempeñar un papel decisivo, tanto a nivel de competitividad y política económica, como de ordenación territorial y de estabilidad en las condiciones del marco geográfico y social de una ciudad global o metropolitana.

La rehabilitación urbana de las áreas de actividad económica debe dar respuesta a las necesidades reales de las empresas, a la transformación y perfeccionamiento permanente de los procesos industriales que se instalan en ellas, pero también ha de asumir los compromisos más amplios de un territorio articulado en base a las demandas de un tiempo y una sociedad. Su modernización tendrá una influencia directa sobre los requerimientos universales de la sostenibilidad, asumidas ya las afecciones directas de cualquier actuación urbanistica sobre un medio, la calidad de vida y las señas de identidad de un territorio.

Es necesario, en ese sentido, afrontar trabajos de carácter analítico y propositivo que sirvan de guia para poder garantizar un proceso de recalificación de estos suelos urbanos que, a menudo, están en condiciones de degradación, de envejecimiento o, en el mejor de los casos, carentes de previsiones a medio y largo plazo que aseguren su pervivencia. Se trata, por tanto, de abordar el tema desde un análisis pormenorizado de aquellos espacios de actividad económica que tienen influencia y determinan la formación de un territorio equilibrado cuyo foco principal de dinamismo recae cada vez más sobre las ciudades de escala metropolitana.
\end{abstract}

Palabras clave: Planificación territorial, Áreas de actividad económica, Área metropolitana, industria, regeneración urbana

\section{Introducción}

Esta comunicación pretende incidir en la necesidad de abordar estudios y propuestas de regeneración, recualificación y renovación de las Áreas de Actividad Económica (AAE) ${ }^{1}$ en un contexto metropolitano y bajo criterios de sostenibilidad y equilibrio territorial.

En las áreas metropolitanas es común observar la proliferación de polígonos industriales localizados en las proximidades de las principales ciudades de referencia, junto a infraestructuras y equipamientos. Son asentamientos que en muchos casos se originaron como la suma de instalaciones particulares sobre una trama rural o en ocasiones también junto a empresas punteras ya en producción, buscando en esta proximidad a ciudad e infraestructuras una localización económicamente ventajosa. Como ya apuntaba Fernando de Terán (Terán,1972), "la prevalencia económica de los "factores de aglomeración" frente a los "factores de dispersión" era, junto con la existencia de 
infraestructuras, una de las varias razones que contribuian eficazmente a producir una concentración espontánea de empresas industriales sobre áreas geográficas limitadas, al mismo tiempo que esta concentración daba lugar a la aparición de "economías externas" que favorecian aún más la implantación industrial en esas áreas." Sin embargo, esta implantación en el territorio no sigue, en la mayoría de los casos, un orden o lógica territorial que derive de un planteamiento y ordenación urbanística de escala local, supramunicipal o regional.

A su vez, la Estrategia Territorial de la Comunitat Valenciana (ETCV, 2011) indica que entender estos procesos metropolitanos "permitirá orientar las áreas metropolitanas hacia patrones que permitan una mayor eficiencia del territorio y un mejor desarrollo e implantación de las actividades económicas." De acuerdo con esto, el Área Metropolitana de Valencia (AMV), considerada como un área en desarrollo de vital importancia en el contexto nacional y europeo, puede servir de modelo como caso de análisis y de estudio propositivo para la mejora del equilibrio territorial y también de la actividad económica, principal motor de desarrollo de las regiones, con los valores cada vez más necesarios de calidad y sostenibilidad.

\section{La evolución de la industria en el Área Metropolitana de Valencia}

La definición formal y material de un proyecto, Si bien el territorio valenciano no fue en los inicios de la industrialización una de las regiones más representativas o pioneras a nivel nacional, sí que al final del siglo XIX se posiciona como la tercera región industrializada del país, tras Cataluña y el País Vasco (Cano, Blasco, 2002). Desde entonces, su localización industrial ha ido variando en función de fuentes de energía, los sistemas de transporte y la normativa urbanística, entre otros muchos factores, hasta ubicarse en entornos metropolitanos.

Cabe destacar la aprobación del Plan General de Ordenación Urbana de Valencia y su Comarca en 1946 (ver figura 1) y la creación de la Corporación Administrativa de la Gran Valencia en 1948, que agrupaba como "su cintura" a 29 municipios vecinos. Este era el primer plan en el que se plasmaron estrategias de escala territorial, con una destacable previsión de suelo industrial y sin límites administrativos municipales dibujados, asumiendo así una visión global $\mathrm{y}$ no de crecimientos individuales aislados. También proponía una descentralización de los nuevos desarrollos, respetando la huerta circundante, limitando el crecimiento de la ciudad de Valencia y favoreciendo un desarrollo en unidades menores articuladas y limitadas por el verde y dentro de una visión regionalista del planeamiento vigente en esos momentos (Pecourt, Piñón, 1997). En este plan destacan, aparte de pequeñas áreas industriales en los municipios, zonas de mayor entidad destinadas a desarrollo industrial apoyadas en las infraestructuras de transporte y también próximas al puerto: carretera de Barcelona y vía férrea al norte, carretera de Madrid y vías férreas de Llíria y Utiel al oeste, el corredor València-Xàtiva hacia el sur, y puerto y zona franca costera al este.

En los años 60, superada la posguerra, se inicia una etapa de reindustrialización que se refleja de forma desigual en el territorio: mientras en los ejes oeste y sur se produce un crecimiento gradual, en el eje norte el desarrollo es más lento debido todavía a la importancia de la agricultura. En 1966, y tras la decisión de desviar el cauce del río Túria, se aprueba el Plan General de Ordenación Urbana de Valencia y su Comarca (figura 1), que, matiza la estrategia territorial del plan del 46, y otorga a la estructura viaria un importante peso territorial y a la industria una gran cantidad de suelo disponible con una modesta regulación urbanística.

A partir de los años 70, la actividad industrial empieza a descentralizarse y a buscar nuevas localizaciones en los municipios colindantes, donde existe abundante oferta de suelo industrial económico por ocupar y junto a las infraestructuras del sistema de radiales y circunvalaciones que el plan había propuesto. Esta planificación, junto con las ordenaciones que los diferentes municipios han hecho en sus términos, es la que en gran medida configuran el suelo actual destinado a las actividades económicas en el Área Metropolitana de 


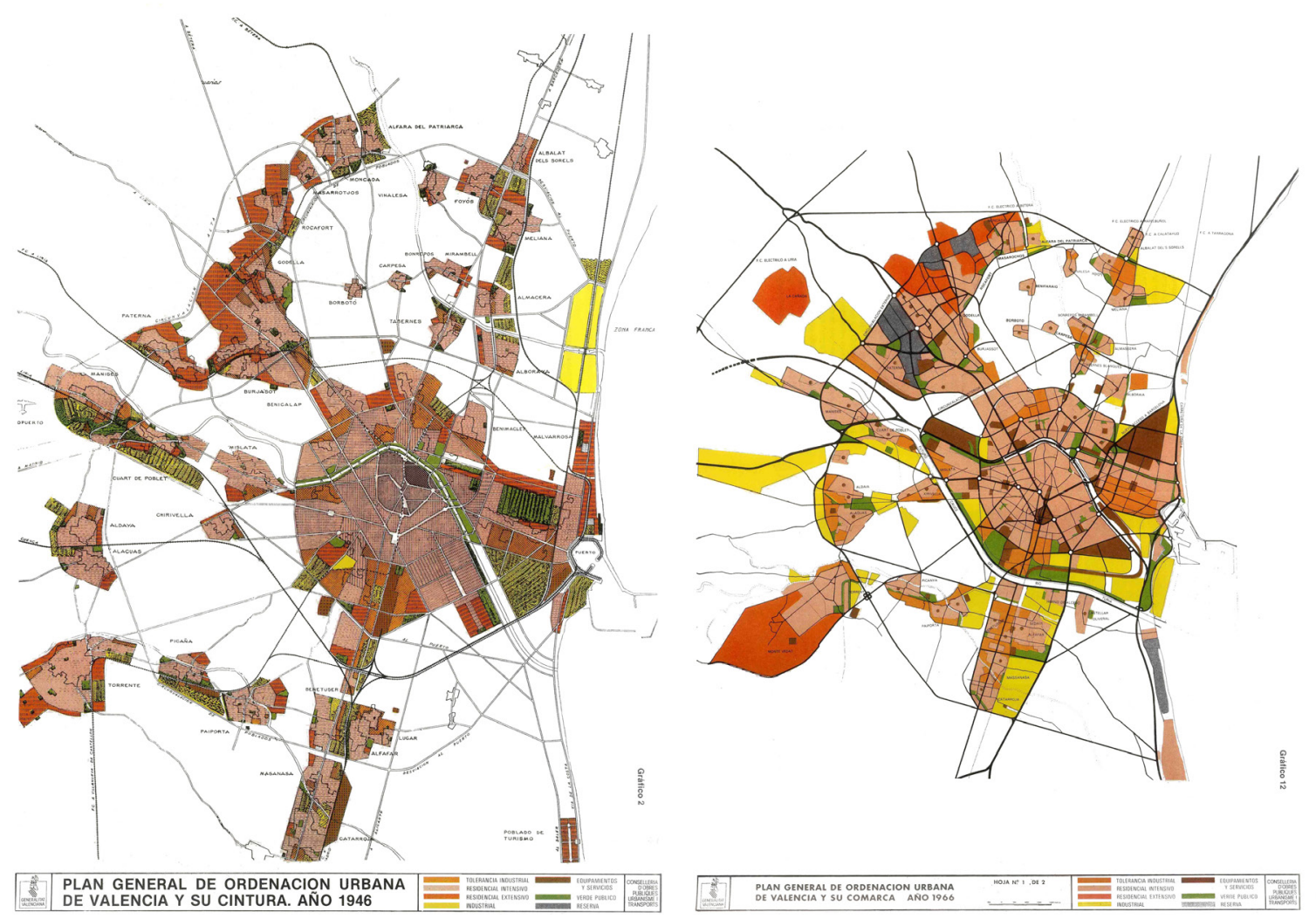

Fig. 1. Izquierda: Plan General de Ordenación Urbana de Valencia y su cintura. Año 1946. Derecha: Plan General de Ordenación Urbana de Valencia y su comarca. Año 1966 (Fuente: COPUT: La Gran Valencia. Trayectoria de un Plan General. Consellería de Obras Públicas Urbanismo y Transportes. Valencia. 1986)

Valencia. Ni el Consell Metropolità de l'Horta, con 44 municipios del primer cinturón de Valencia, creado en el 86 con vocación urbanística y medioambiental de carácter territorial, ni las Normas de Coordinación Metropolitana de 1988, que regulaba los crecimientos urbanísticos del AMV, tuvieron el margen de actuación supramunicipal para el que fueron creados. Fue la Generalitat Valenciana la que asumiría las competencias territoriales y los municipios, los planeamientos locales.

A partir de los años 80 se produce una descentralización y dispersión de la residencia y de las actividades económicas sobre los principales ejes viarios, al noroeste, sobre la A3 y sobre la Pista de Silla al sur. Esta fuerte expansión suburbana del AMV continúa y se acentúa más en la segunda mitad de los 90 sobre un territorio sin modelo metropolitano (Ponce, 2013), siendo el resultado el marco urbano $y$ territorial sobre el que nos encontramos actualmente. El resultado en la actualidad es una especialización terciaria de la ciudad de
Valencia, siendo la primera y segunda corona las que mantienen en cierta medida el uso industrial $\mathrm{o}$, como veremos más adelante, los servicios a la industria. Como apunta Salom (Salom, 2015), la estructura territorial resultante del AMV ha experimentado una importante transformación en los últimos años, pasando del clásico modelo radial a una pauta territorial de una mayor complejidad. La movilidad radial sigue manteniendo su importancia, pero la descentralización y dispersión de la residencia y la evolución de la actividad económica ha provocado un cambio en los flujos y en las relaciones entre usos urbanos.

\section{Las áreas de actividad económica del Área Metropolitana de Valencia}

Si bien en España no existe una delimitación oficial de las áreas metropolitanas, sí que existen variables relacionadas con la movilidad residencia-trabajo para establecer delimitaciones en torno a cada ciudad. El 
término Área Metropolitana es utilizado por el Instituto Geográfico Nacional para "describir la nueva forma de la ciudad actual, en la que el espacioconstruidoy, con ello, el de las funciones y flujos supera los limites tradicionales, físicos y administrativos de la ciudad tradicional para abarcar territorios cada vez más extensos. Por encima de su potencial plasmación jurídicopolítica, es el nuevo espacio de vida, colectivo y compartido, del ciudadano contemporáneo. Reconocer esta realidad no es solo un mero objetivo académico, estadístico o territorial, sino también una necesidad para adecuar numerosas políticas públicas a la escala real en la que estas requieren ser abordadas. Significativamente, el Instituto Nacional de Estadística (INE) ha empezado a publicar, dentro del proyecto europeo Urban "Áreas Urbanas Funcionales" Audit, indicadores urbanos para las españolas delimitadas a partir de dicha variable."2

En el caso de Valencia, la delimitación del área metropolitana establecida por el ayuntamiento ${ }^{3}$, coincide en gran medida con las infraestructuras viarias que han propiciado y condicionado la localización de la industria junto a las principales vías radiales y con la delimitación de la A7. Como se observa en la figura 2, dicho ámbito dispone de una cantidad importante de suelo urbano calificado como industrial y terciario. También se observa la concentración de estas áreas en las proximidades a la ciudad central y de las vías radiales (ejes norte, noroeste, oeste y sur), siendo menor la presencia de asentamientos en zonas interiores respecto de la A-7. Esto muestra la relación ciudad-industria actual ya que "la industria continúa siendo una actividad importante en la mayoría de las grandes urbes y las aglomeraciones desarrolladas en su entorno" (Caravaca, Méndez, 2003). Sin embargo, como se ha indicado y se puede apreciar en el plano, la expansión territorial de las AAE producida en los últimos veinte años ha seguido unos criterios en los que el territorio se ha desarrollado sin ninguna directriz supramunicipal y sustentado en el enriquecimiento local e individual de cada municipio (Cano et al. 2018).

Además, si ampliamos la visión territorial del AMV y atendemos a la estructura de cada uno de estos asentamientos observamos que en muchas ocasiones su ordenación tampoco ha seguido modelos urbanísticos claros. Son numerosos los casos en los que se puede apreciar trazos de parcelario agrícola en polígonos industriales


Fig. 2. Izquierda: Delimitación del AMV del Ayuntamiento de Valencia y relación con las principales infraestructuras viarias. Derecha: Suelo industrial y terciario del entorno de Valencia. (Fuente: elaboración propia a partir de datos del Institut Cartogràfic Valencià y Cátedra Divalterra 2018) 
consolidados, o incluso naves industriales en suelo rural, con escasa regulación y muchas veces con una importante carencia de calidad urbana y de servicios mínimos. Esas primeras industrias, establecidas de forma espontánea, han acabado siendo asumidas como suelo urbano. La suma de muchos casos como este, junto con el polígono industrial al que cada municipio ha destinado suelo, ha producido el salpicado territorial de decenas de polígonos y asentamientos industriales con escasa jerarquía territorial y sin un marco supramunicipal.

El hecho de que en la provincia de Valencia un porcentaje elevado de áreas industriales se haya desarrollado de forma espontánea y sin articulación (Martínez, 2003) propicia una ocupación menos eficiente del territorio, afecciones medioambientales importantes y una menor optimización de los recursos, de los servicios e infraestructuras o de la movilidad. Suponen, por tanto, un impacto que va más allá de los límites municipales donde se han posicionado. Por ello, es evidente que el estudio de las áreas de actividad económica no puede realizarse de forma aislada. Es necesaria una visión territorial en conjunto, sin límites administrativos, que corrija, reconduzca y facilite la actividad económica bajo las nuevas exigencias tanto de las empresas como de los nuevos objetivos de desarrollo sostenible.

\section{Los retos de las AAE en la AMV. Nuevas necesidades}

Como hemos visto, la evolución de la industria y su implantación en el territorio en nuestro país y en el AMV en concreto ha seguido unas dinámicas muy alejadas de planificaciones metropolitanas. Actualmente, la actividad económica sigue buscando estas localizaciones, pero evoluciona a más velocidad que los planteamientos urbanísticos y territoriales que la ordenan y localizan en el territorio. En la tendencia general para las área metropolitanas, según Font (Font, 2015), se produce una "nueva metropolización": “ frente a la industria como motor principal de crecimiento en aquel largo periodo del anterior proceso de urbanización, la lógica dominante de lograr economías de escala y, el resultado de un territorio fuertemente jerarquizado y una ciudad compacta y centrípeta, el territorio actual es consecuencia de un cambio generalizado en la estructura económica de la sociedad, en la que los servicios pasan a ser el nuevo motor principal del crecimiento, la producción es segmentada y flexible y la ciudad centrifuga actividades y flujos por el territorio, constituyendo unos sistemas metropolitanos discontinuos y de jerarquías blandas."

Además, ya no nos encontramos ante un problema de oferta de suelo disponible, sino de calidad del mismo. A estructuras urbanas desfasadas hay que añadirle el cambio de actividad que en ellas se está produciendo, debido a la creciente terciarización de la industria, en la que cobran importancia tareas de gestión, planificación, diseño, logística, comercialización y servicios en general. No estamos, por tanto, ante la necesidad de polígonos industriales tradicionales, ya que las actividades que se dan en ellos ya no se corresponden con la industria tradicional productiva, sino que se ha ampliado el abanico de actividades económicas allí desarrolladas.

También sabemos que el consumo desmesurado años atrás del recurso suelo debe llegar a su fin, proponiendo nuevos modelos de regeneración de suelo urbano existente. La rehabilitación urbana de las áreas de actividad económica debe dar respuesta a las nuevas necesidades de las empresas, a la transformación y perfeccionamiento permanente de los procesos industriales que se instalan en ellas, pero también ha de asumir los compromisos más amplios de un territorio articulado y equilibrado en base a las demandas de un tiempo y una sociedad, tal y como indican las políticas europeas en los últimos años.

Ante ese reto de escala territorial, hay que destacar que, en la Comunitat Valenciana, las nuevas directrices y regulaciones urbanísticas, tras el proceso de liberación del suelo de los años 90 (ETCV, Ley de Ordenación del Territorio, Urbanismo y Paisaje - LOTUP- y su posterior revisión, o los diferentes Planes de Acción Territorial) han abierto un nuevo marco de trabajo de carácter supramunicipal en los últimos años. Además, la adhesión al Convenio Europeo del Paisaje y su incorporación a la legislación autonómica han propiciado una serie de planes supramunicipales que abren 
un nuevo horizonte en el que el territorio asume un papel importante en su gestión, ordenación y desarrollo. En los planteamientos encaminados a mejorar y articular los espacios de actividad económica, se parte de favorecer el crecimiento económico dentro de un sistema territorial articulado, en coherencia con la infraestructura verde (figura 3), y las diferentes relaciones urbanas.

La solución, por tanto, no pasa sólo por la revisión individual de cada uno de los polígonos industriales actuales, sino que necesita un análisis general del tejido productivo actual. En este sentido, el ámbito de un área metropolitana, como el AMV, cobra más importancia si cabe, puesto que se requieren soluciones amplias que pasen por una revisión de las jerarquías territoriales, de las compatibilidades de usos del suelo, de la movilidad, de las relaciones residencia-empleo, de los flujos de mercancías e información y de las propias instalaciones, entre otros muchos factores.

\section{La recomposición del espacio de actividad económica. Líneas de actuación}

Como vemos, nos encontramos ante un escenario industrial generalmente obsoleto, que todavía se rige por las dinámicas y planeamientos de años atrás, y unas necesidades por parte de las nuevas industrias y economías, que demandan nuevas condiciones para el desarrollo de su actividad. En este punto, cabe destacar la delimitación real y operativa que nos pueden ofrecer las áreas metropolitanas, mediante el análisis, la interpretación de su estado actual y evolución.

En ese sentido, es importante realizar un análisis pormenorizado y sistematizado de la situación actual, tanto cuantitativo como cualitativo, y a diferentes escalas, que permita entender la naturaleza y evolución de estas áreas tanto individualmente como en relación con su entorno metropolitano. También es significativo reconocer en ellas las posibilidades y expectativas de futuro a medio y largo plazo y posicionarlas en el sistema articulado y policéntrico de su territorio. Esto permitirá reconocer sus potencialidades y limitaciones de cara a su recomposición. En el AMV, ingredientes como el puerto, el aeropuerto, el futuro corredor mediterráneo y sus zonas logísticas, entre otros, ponen de manifiesto el sistema policéntrico ante el que nos encontramos y las diferentes oportunidades posibles.

Pero también es valioso que el análisis sea propositivo para así lanzar unas líneas o estrategias de actuación que guíen y acoten los procesos de intervención (figura 4). Como hemos visto, los planteamientos territoriales de las administraciones locales y las organizaciones europeas ponen en un primer estadio los compromisos medioambientales y ecológicos, así como los valores paisajísticos y culturales de cada lugar. El territorio y la infraestructura verde, por tanto, deben ser los primeros ordenadores de las actividades urbanas sin menoscabo de la competitividad económica que aportan estas dinámicas

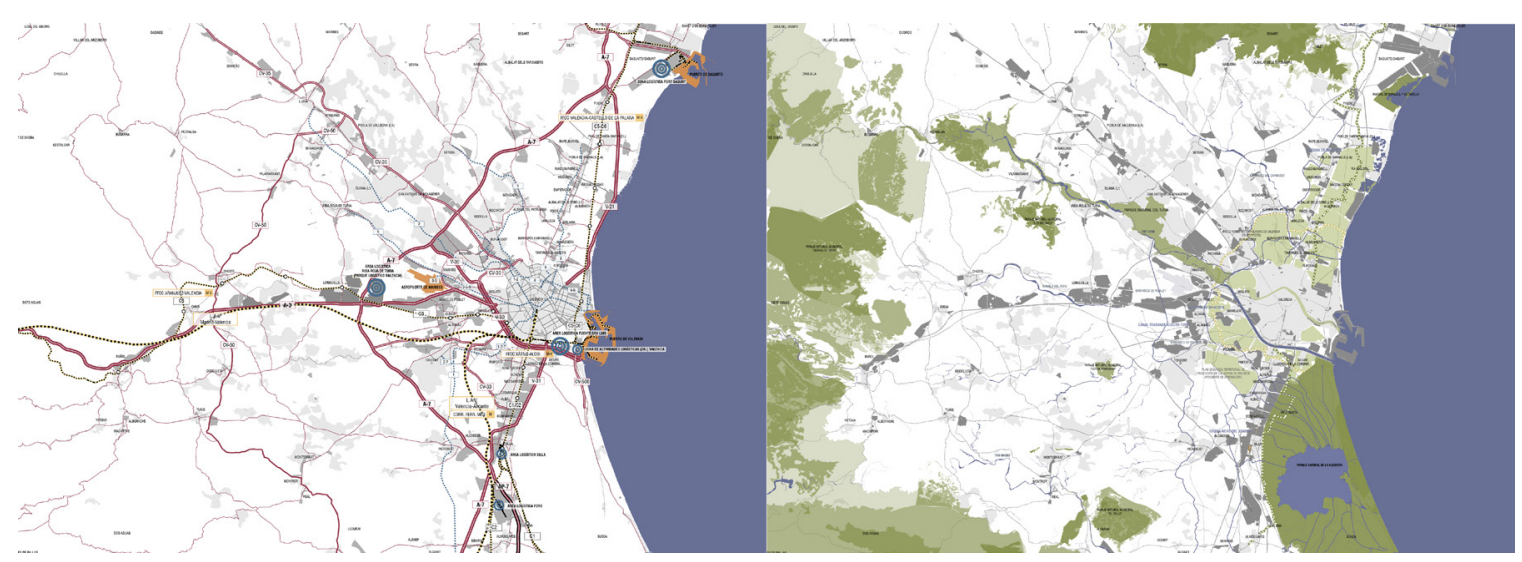

Fig. 3. Infraestructuras de transporte (izquierda) e infraestructura verde (derecha) del entorno de Valencia. (Fuente: Cátedra Divalterra, 2018) 


\section{empresariales.}

Otra línea por desarrollar será la movilidad, la proximidad y la accesibilidad territorial. Como hemos visto, las infraestructuras viarias y ferroviarias, sobre todo las de primer orden, han servido de canales del crecimiento industrial espontáneo. Tanto estos ejes como sus accesos son el soporte de los flujos de su actividad, por lo que la articulación y la relación jerárquica con el resto de infraestructuras será clave a la hora de posicionar cada una de las AAE en el contexto metropolitano. En esta línea, también es de vital importancia la consideración del transporte público y la movilidad blanda en la cohesión territorial de las áreas metropolitanas. En el AMV destacan las conexiones radiales con el centro de la ciudad, pero sigue existiendo una importante carencia de infraestructuras que articulen esta nueva realidad policéntrica más compleja.

En cuanto a las actividades económicas, hemos visto que la actividad industrial productiva del siglo pasado ha evolucionado a otras actividades con unas externalidades mucho menos nocivas y con otras necesidades materiales e inmateriales. En este sentido, los términos "industrial" y "terciario" de los planeamientos municipales ofrecen un mapa industrial del AMV plano y obsoleto que no responde a la realidad del funcionamiento de estas áreas. Por ello es necesaria una reflexión sobre los usos del suelo, la compatibilidad y complementariedad entre ellos, incluso con el residencial, los cambios de tipologías arquitectónicas o la calidad del espacio público, que permitan una mayor flexibilidad a esta diversificación de la actividad económica. También es creciente el interés por mejorar la imagen de la empresa, aumentando la exigencia de calidad ambiental como factor diferencial de unos emplazamientos con respecto a otros. Por tanto, el estudio de las condiciones de imagen, de la calidad del espacio público y de la visibilidad de estas áreas es otra estrategia importante a la hora de recomponer estos espacios. (figuras 5 y 6 )

\section{Conclusiones}

Las áreas de actividad económica desempeñan un papel fundamental en el desarrollo económico de una región y su localización en el territorio ha sido clave para su progreso. Sin embargo, la evolución de la actividad económica y las nuevas necesidades que plantean requieren, desde la disciplina urbanística, de una reflexión de los factores de competitividad con unos nuevos compromisos territoriales y ambientales.

Los tejidos urbanos pueden favorecer la continuidad de la actividad económica, o bien, si no se actúa, pueden llegar a impedirlo. Por ello es necesario no abordar soluciones individuales para cada polígono, sino contextualizarlos en un marco territorial más amplio, como la escala metropolitana, de manera que se puedan plantear estrategias amplias e integradoras, con objetivos a medio y largo plazo. Las administraciones juegan aquí un papel fundamental, ya que deben ser

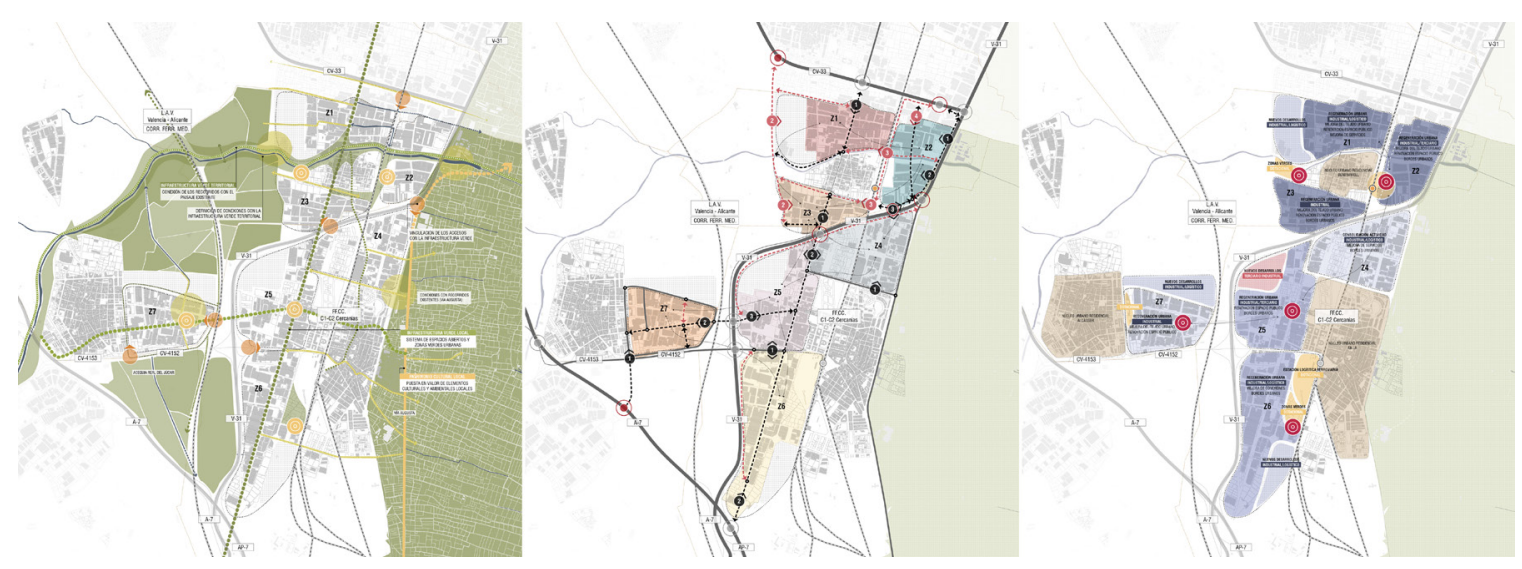

Fig. 4. Estrategias de intervención en las áreas industriales del entorno de la Pista de Silla (AMV). De izquierda a derecha: infraestructura verde, accesos territoriales y regeneración urbana. (Fuente: Cátedra Divalterra, 2018) 


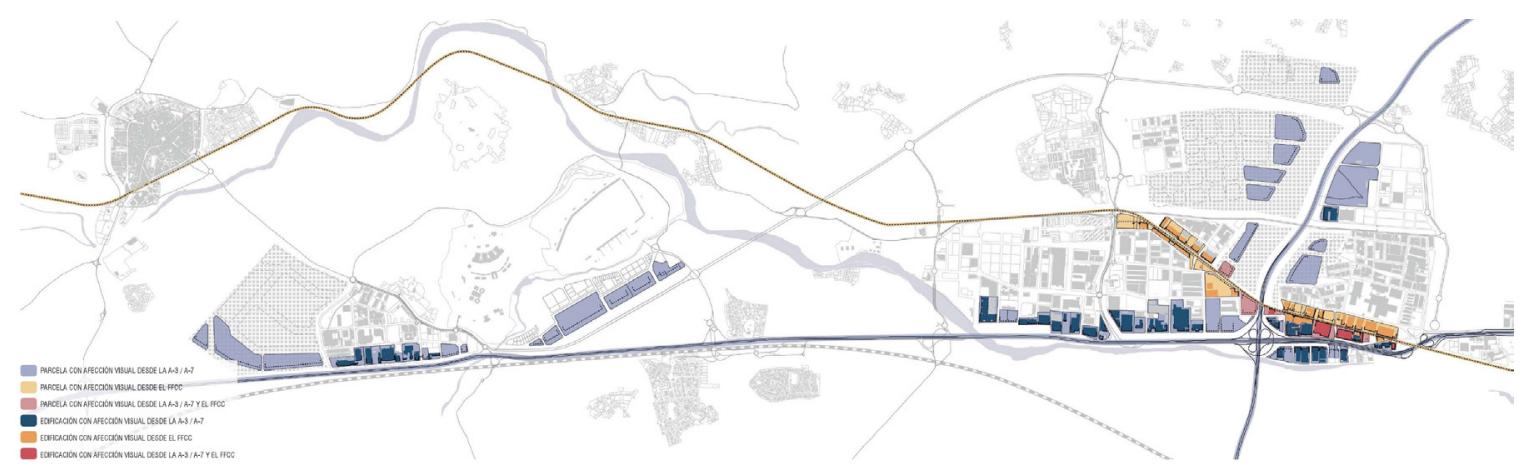

Fig. 5. Estudio de visibilidad de parcelas y edificios industriales desde las infraestructuras en el entorno de la A3 y A7. (Fuente: Cátedra Divalterra, 2018)

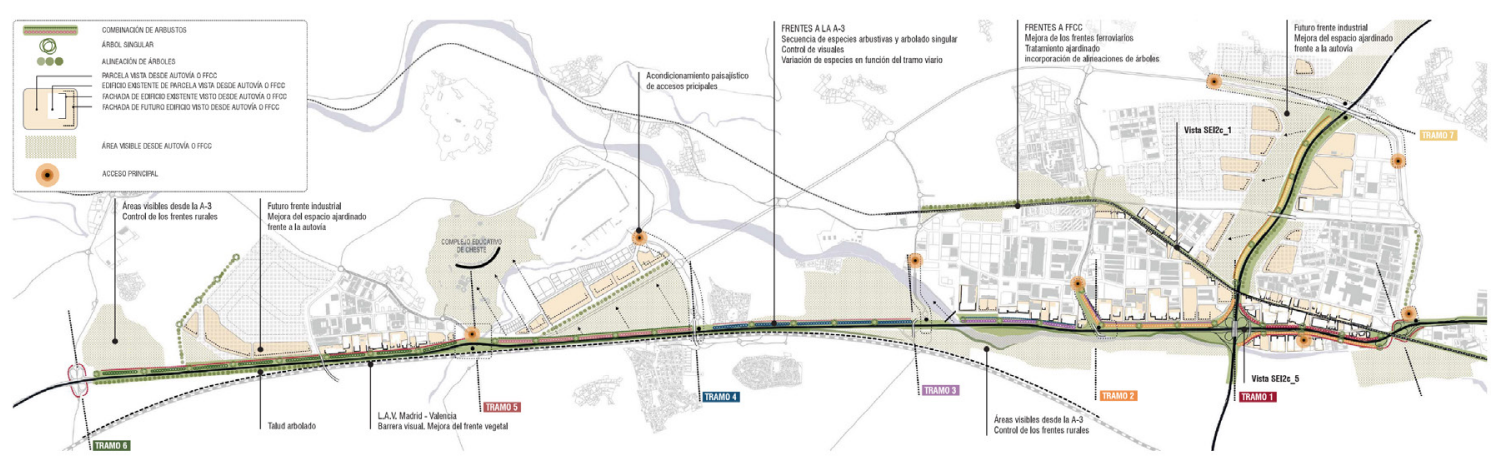

Fig. 6. Propuesta de tratamiento del espacio público en los ejes viarios y ferroviarios en el entorno de la A3 y A7. (Fuente: Cátedra Divalterra, 2018)

las impulsoras de este análisis que identifique las tendencias en curso, reconduzca la situación actual y favorezca la actividad económica en el marco de los nuevos objetivos de desarrollo sostenible.

Además, un planteamiento de la regeneración de estas áreas económicas a partir de un análisis global puede establecer diferentes prioridades y modelos de intervención, en función de las diferentes tipologías, grados de obsolescencia o nuevos requisitos de las AAE, que permitan una optimización del reparto de recursos económicos destinados a la mejora de estos espacios en un contexto territorial articulado y equilibrado. En este sentido, en el AMV, los nuevos compromisos con la cultura del territorio abren un nuevo periodo de oportunidades y de formas respetuosas de intervenir que los urbanistas hemos de saber orientar desde nuevos modelos de análisis e intervención.

\section{Notas}

1 AAE: Un área de actividad económica, según la Coordinadora Española de Polígonos Españoles (CEPE), es un espacio urbano consolidado, desarrollado para la instalación de actividades económicas variadas, constituyendo una zona perfectamente planificada y delimitada, con ordenanzas y espacios dotacionales y con unos estándares urbanísticos e infraestructuras acordes con los requisitos establecidos en las leyes urbanísticas vigentes y reglamentos correspondientes.

2 Instituto Geográfico Nacional en "España en Mapas. Una síntesis geográfica". Madrid, 2018.

3 En el caso de Valencia, su delimitación de Área Metropolitana ha ido variando a lo largo del siglo XX en función de diferentes organismos y estudios. Actualmente el área está formada por los 44 municipios de las comarcas Horta Nord, Horta Oest, Horta Sud y València (Ayuntamiento de Valencia, 2018)

\section{Referencias}

Cano Hurtado, Juan J., Blasco Sánchez, Carmen, Martínez Pérez, Francisco J., 
Gascón Hernández, Ana, y Esteve Aguado, Carlos. 2018. Renovación urbana y actividad económica. Propuesta de intervención territorial en un enclave logístico. Valencia: Editorial Universitat Politècnica de València. Martínez Pérez, Francisco J., Blasco Sánchez, Carmen, Cano Hurtado, Juan J., Escoms Martínez, Anna, Gascón Hernández, Ana, Deltoro Soto, Julia. 2016. Territorio y actividades económicas. La provincia de Valencia frente al reto del Corredor Ferroviario Mediterráneo. Valencia: Editorial Universitat Politècnica de València.

Font Arellano, Antonio. 2015. Territorios urbanos. Memoria de una búsqueda apasionada. Barcelona: Iniciativa Digital Politècnica.

Salom Carrasco, Julia, 2015. "Crisis y reestructuración en el Área Metropolitana de Valencia". Revista de Estudios Andaluces 32: 1-21.

Ponce Herrero, Gabino. 2013. "Estrategias de metropolización de la ciudad de Valencia en la etapa autonómica: La centralidad cuestionada en el modelo Postchristaller de ordenación del Territorio". Boletín de la Asociación de Geógrafos Españoles", 62: 147-172.

Font Arellano, Antonio, Vecslir Peri, Lorena, Maristany Jackson, Lorena, Mas Artigas, Sílvia. 2012. Patrons urbanístics de les activitats econòmiques: regió metropolitana de Barcelona. Barcelona: Institut d'Estudis Territorials, Generalitat de Catalunya.

Nel·lo i Colom, Oriol. 2012. Ordenar el territorio: la experiencia de Barcelona y Cataluña. Valencia: Valencia: Tirant humanidades, 2012.

López Groh, Francisco. 2011. La regeneración de áreas industriales. Madrid: Sepes Entidad Estatal de Suelo.

Font Arellano, Antonio y Vecslir Peri, Lorena. 2008. «Nuevas geografías de la producción y el consumo en la Región Metopolitana de Barcelona». Scripta Nova. Revista Electrónica de Geografía y Ciencias Sociales, 12 (270).

Institut Catalá del Sòl. 2007. Sectores de Actividad Económica. Libro de estilo. Barcelona: Institut Catalá del Sòl.

Esteban I Noguera, Juli. 2007. «Els espais d'activitats econòmiques en el planejament territorial». Papers: Regió Metropolitana de Barcelona: Territori, estratègies, planejament, 45, 56-63.

Caravaca, Inmaculada y Méndez, Ricardo. 2003. «Trayectorias industriales metropolitanas: nuevos procesos, nuevos contrastes». Eure, 12 (87), 37-50.

Cano Hurtado, Juan J., Blasco Sánchez, Carmen. 2002. El Espacio Industrial en la Comunitat Valenciana. Valencia: Editorial Universitat Politécnica de València.

de Terán, Fernando, 1972. "Descongestión industrial, zonificación y urbanismo: Experiencias extranjeras y en España”. En 3 Congreso Nacional Iranor: Dirección de empresas y cambios tecnológicos. Madrid, (23-36). 